Publ. Mat. 60 (2016), 27-53

DOI: 10.5565/PUBLMAT_60116_02

\title{
A NONLOCAL 1-LAPLACIAN PROBLEM AND MEDIAN VALUES
}

\author{
José M. Mazón, Mayte PÉrez-Llanos, Julio D. Rossi, \\ AND JULián TOLEDO
}

\begin{abstract}
In this paper, we study solutions to a nonlocal 1-Laplacian equation given by

$$
-\int_{\Omega_{J}} J(x-y) \frac{u_{\psi}(y)-u(x)}{\left|u_{\psi}(y)-u(x)\right|} d y=0 \quad \text { for } x \in \Omega,
$$

with $u(x)=\psi(x)$ for $x \in \Omega_{J} \backslash \bar{\Omega}$. We introduce two notions of solution and prove that the weaker of the two concepts is equivalent to a nonlocal median value property, where the median is determined by a measure related to $J$. We also show that solutions in the stronger sense are nonlocal analogues of local least gradient functions, in the sense that they minimize a nonlocal functional. In addition, we prove that solutions in the stronger sense converge to least gradient solutions when the kernel $J$ is appropriately rescaled.
\end{abstract}

2010 Mathematics Subject Classification: 45G10, 45J05, 47H06.

Key words: 1-Laplacian, median value, least gradient functions.

\section{Introduction}

A well known fact is that solutions to some partial differential equations are related to mean value properties. A classical example that one can find in any elementary PDE textbook states that $u$ is harmonic in a domain $\Omega \subset \mathbb{R}^{N}$ (that is, $u$ verifies $\Delta u=0$ in $\Omega$ ) if and only if it verifies the mean value property

$$
u(x)=\frac{1}{\left|B_{\varepsilon}(x)\right|} \int_{B_{\varepsilon}(x)} u(y) d y,
$$

for all $\varepsilon>0$ such that $B_{\varepsilon}(x) \subset \Omega$. In fact, one can relax this condition by requiring that it holds asymptotically

$$
u(x)=\frac{1}{\left|B_{\varepsilon}(x)\right|} \int_{B_{\varepsilon}(x)} u(y) d y+o\left(\varepsilon^{2}\right),
$$

as $\varepsilon \rightarrow 0$. This follows easily for $C^{2}$ functions by using the Taylor expansion and for continuous functions by using the theory of viscosity 
solutions. A weak asymptotic mean value formula holds in some nonlinear cases as well. In fact, in [8] the authors characterize $p$-harmonic functions, that is, solutions to the $p$-Laplacian,

$$
\Delta_{p} u=\operatorname{div}\left(|\nabla u|^{p-2} \nabla u\right)=0,
$$

for $1<p \leq \infty$, by means of an asymptotic mean value property.

In $[7]$ the authors characterize solutions to the following problem

$$
\Delta_{1}^{H} u=0
$$

in terms of another asymptotic geometric property; the operator $\Delta_{1}^{H}$ is given by

$$
\Delta_{1}^{H} u:=|D u| \operatorname{div}\left(\frac{D u}{|D u|}\right),
$$

which is a variant of the 1-Laplacian given by $\operatorname{div}\left(\frac{D u}{|D u|}\right)$. They show, in the two dimensional case, the asymptotic expansion

$$
u(x)-\operatorname{median}_{s \in \partial B_{\varepsilon}(x)} u(s)=-\frac{\varepsilon^{2}}{2} \Delta_{1}^{H} u(x)+o\left(\varepsilon^{2}\right) ;
$$

here, the median of a continuous function over a measurable set $A$, $\operatorname{median}_{s \in A} u(s)=m$, is defined as the unique value $m$ such that, for $\mu$ the 1-dimensional Hausdorff measure,

$$
\mu(\{x \in A: u(x) \geq m\}) \geq \frac{\mu(A)}{2} \quad \text { and } \quad \mu(\{x \in A: u(x) \leq m\}) \geq \frac{\mu(A)}{2} .
$$

Observe that for such definition we just need $u$ to be measurable with respect to $\mu$. However, we just have uniqueness of the value $m$ for continuous functions, see for instance [10]. The relation between the median property and solutions to $\Delta_{1}^{H} u=0$ is further investigated in [12]. In this work the authors prove that functions verifying the following local median value property

$$
\begin{aligned}
u(x)=\operatorname{median}_{s \in \partial B_{r}(x)} u(s), \text { for every } x & \in \Omega \\
& \text { and every } 0<r \leq R(x) \leq \operatorname{dist}(x, \partial \Omega),
\end{aligned}
$$

are solutions to $\Delta_{1}^{H} u=0$ in viscosity sense. This proof is based on the previous asymptotic expansion, thus they restrict themselves to the bidimensional case. Furthermore, if $\Omega \subset \mathbb{R}^{2}$ is a strictly convex bounded open set, they show existence of at least one solution to the Dirichlet problem associated to (1.2), for boundary data $g: \partial \Omega \rightarrow \mathbb{R}$ whose level sets verify adequate conditions. See also [11] for a study of the associated evolution problem. 
On the other hand, in [9] it is proved that the Dirichlet problem for the 1-Laplacian operator

$$
\begin{cases}-\operatorname{div}\left(\frac{D u}{|D u|}\right)=0, & \text { in } \Omega, \\ u=h, & \text { on } \partial \Omega,\end{cases}
$$

has a solution $u \in B V(\Omega)$ for every $h \in L^{1}(\partial \Omega)$. The relaxed energy functional associated to problem (1.3) is the functional $\Phi_{h}: L^{\frac{N}{N-1}}(\Omega) \rightarrow$ $(-\infty,+\infty]$ defined by

$$
\Phi_{h}(u)= \begin{cases}\int_{\Omega}|D u|+\int_{\partial \Omega}|u-h| d \mathcal{H}^{N-1} & \text { if } u \in B V(\Omega), \\ +\infty & \text { if } u \in L^{\frac{N}{N-1}}(\Omega) \backslash B V(\Omega) .\end{cases}
$$

In [9] it is shown that the solutions of problem (1.3) coincide with the functions of least gradient that appear in the theory of parametric minimal surfaces, see $[\mathbf{6}, \mathbf{1 3}, \mathbf{1 4}]$. This problem is quite different from (1.1) since it involves giving a meaning to $\frac{\nabla u}{|\nabla u|}$ when the gradient vanishes. These difficulties were tackled in $[\mathbf{1}]$ (see also [9]) by means of a bounded vector field $z$ which plays the role of $\frac{D u}{|D u|}$. Moreover there are extra difficulties for the Dirichlet boundary condition, which has to be considered in a weak sense.

Our aim here is to study solutions to the nonlocal 1-Laplacian with Dirichlet boundary condition $\psi$ :

$$
\begin{cases}-\int_{\Omega_{J}} J(x-y) \frac{u_{\psi}(y)-u(x)}{\left|u_{\psi}(y)-u(x)\right|} d y=0, & x \in \Omega, \\ u(x)=\psi(x), & x \in \Omega_{J} \backslash \bar{\Omega},\end{cases}
$$

and to relate them with a nonlocal median value property and with a kind of nonlocal least gradient functions. Hereafter, $\Omega \subset \mathbb{R}^{N}$ is a bounded and smooth domain and $J: \mathbb{R}^{N} \rightarrow \mathbb{R}$ a continuous nonnegative radial function, compactly supported in $B_{1}(0)$ with $J(0)>0$, verifying $\int_{\mathbb{R}^{N}} J(z) d z=1$. We denote by

$$
\Omega_{J}=\Omega+\operatorname{supp}(J) \quad \text { and by } \quad u_{\psi}:=u \chi_{\Omega}+\psi \chi_{\Omega_{J} \backslash \bar{\Omega}} .
$$

Let us define the following measure of a set $E \subset B_{1}(0)$ :

$$
\mu_{J}^{0}(E):=\int_{E} J(z) d z .
$$


Therefore, for $f: \mathbb{R}^{N} \rightarrow \mathbb{R}$ a measurable function (not necessarily continuous), a median value $m$ of $f$ with respect to $\mu_{J}^{0}$ is given by:

$$
\mu_{J}^{0}\left(\left\{y \in B_{1}(0): f(y) \geq m\right\}\right) \geq \frac{1}{2} \quad \text { and } \quad \mu_{J}^{0}\left(\left\{y \in B_{1}(0): f(y) \leq m\right\}\right) \geq \frac{1}{2} \text {. }
$$

We denote such fact by

$$
m \in \operatorname{median}_{\mu_{J}^{0}} f .
$$

Also we will denote by sign the multivalued sign-function defined as

$$
\operatorname{sign}(z)= \begin{cases}1 & \text { if } z>0 \\ {[-1,1]} & \text { if } z=0 \\ -1 & \text { if } z<0\end{cases}
$$

With these notations let us introduce our definition of a weak solution to $(1.5)$.

Definition 1.1. Let $\psi \in L^{1}\left(\Omega_{J} \backslash \bar{\Omega}\right)$. We say that $u \in L^{1}(\Omega)$ is a weak solution to (1.5) if there exists $g: \Omega_{J} \times \Omega_{J} \rightarrow \mathbb{R}$ such that $g \in$ $L^{\infty}\left(\Omega_{J} \times \Omega_{J}\right)$ with $\|g\|_{\infty} \leq 1$,

$$
\begin{array}{r}
J(x-y) g(x, y) \in J(x-y) \operatorname{sign}\left(u_{\psi}(y)-u_{\psi}(x)\right), \\
\text { a.e. }(x, y) \in \Omega_{J} \times \Omega_{J},
\end{array}
$$

and

$$
-\int_{\Omega_{J}} J(x-y) g(x, y) d y=0, \quad \text { a.e. } x \in \Omega .
$$

We have the following characterization of weak solutions of the nonlocal 1-Laplacian with Dirichlet boundary condition in terms of a nonlocal median value property.

Theorem 1.2. Given $\psi \in L^{1}\left(\Omega_{J} \backslash \bar{\Omega}\right)$, we have that $u$ is a weak solution to (1.5) with Dirichlet datum $\psi$ if and only if, $u$ verifies the following nonlocal median value property:

$$
u(x) \in \operatorname{median}_{\mu_{J}^{0}} u_{\psi}(x-\cdot), \quad x \in \Omega,
$$

that is, for $x \in \Omega$,

$$
\begin{aligned}
& \mu_{J}^{x}\left(\left\{y \in B_{1}(x): u_{\psi}(y) \geq u(x)\right\}\right) \geq \frac{1}{2} \quad \text { and } \\
& \mu_{J}^{x}\left(\left\{y \in B_{1}(x): u_{\psi}(y) \leq u(x)\right\}\right) \geq \frac{1}{2},
\end{aligned}
$$

where $\mu_{J}^{x}(E):=\int_{E} J(x-y) d y$ for $E \subset B_{1}(x)$. 
If in addition, we assume in Definition 1.1 that the function $g$ is antisymmetric, we get a more restrictive concept of solution, which we call variational solution since it can be characterized as a minimizer of the functional $\mathcal{J}_{\psi}: L^{1}(\Omega) \rightarrow[0,+\infty[$ given by

$$
\mathcal{J}_{\psi}(u):=\frac{1}{2} \int_{\Omega_{J}} \int_{\Omega_{J}} J(x-y)\left|u_{\psi}(y)-u_{\psi}(x)\right| d x d y .
$$

This functional $\mathcal{J}_{\psi}$ is the nonlocal version of the energy functional $\Phi_{h}$ defined by (1.4)

Definition 1.3. Let $\psi \in L^{1}\left(\Omega_{J} \backslash \bar{\Omega}\right)$. We say that $u \in L^{1}(\Omega)$ is a variational solution to (1.5) if there exists $g: \Omega_{J} \times \Omega_{J} \rightarrow \mathbb{R}$ such that $g \in L^{\infty}\left(\Omega_{J} \times \Omega_{J}\right)$ with $\|g\|_{\infty} \leq 1$ verifying

$$
\begin{array}{r}
J(x-y) g(x, y) \in J(x-y) \operatorname{sign}\left(u_{\psi}(y)-u_{\psi}(x)\right), \\
\text { a.e. }(x, y) \in \Omega_{J} \times \Omega_{J},
\end{array}
$$

and

$$
-\int_{\Omega_{J}} J(x-y) g(x, y) d y=0, \quad \text { a.e. } x \in \Omega .
$$

Obviously any variational solution is a weak solution for the nonlocal 1-Laplacian, but we will show in Subsection 3.1 that the class of variational solutions is strictly smaller than the class of weak solutions.

Theorem 1.4. Let $\psi \in L^{1}\left(\Omega_{J} \backslash \bar{\Omega}\right)$. Then $u \in L^{1}(\Omega)$ is a variational solution to (1.5) if and only if it is a minimizer of the functional $\mathcal{J}_{\psi}$ given in (1.9).

The following result links nonlocal with local problems (see also [2, 4]):

Theorem 1.5. Let $\Omega$ be a smooth bounded domain in $\mathbb{R}^{N}$ and $\tilde{\psi} \in$ $L^{\infty}(\partial \Omega)$. Take a function $\psi \in W^{1,1}\left(\Omega_{J} \backslash \bar{\Omega}\right) \cap L^{\infty}\left(\Omega_{J} \backslash \bar{\Omega}\right)$ such that $\left.\psi\right|_{\partial \Omega}=\tilde{\psi}$. Assume also $J(x) \geq J(y)$ if $|x| \leq|y|$. Let $u_{\varepsilon}$ be a variational solution to (1.5) for $J_{\varepsilon}(x):=\frac{1}{\varepsilon^{N+1}} J\left(\frac{x}{\varepsilon}\right)$. Then, up to a subsequence,

$$
u_{\varepsilon} \rightarrow u \quad \text { in } \quad L^{1}(\Omega)
$$

being $u$ a solution to (1.3) with $h=\tilde{\psi}$. 
To conclude this introduction we would like to mention that in [2] (see also [4]) the authors study the evolution problem for a nonlocal operator with Dirichlet boundary conditions:

$$
u_{t}(x, t)=\int J(x-y)|u(y, t)-u(x, t)|^{p-2}(u(y, t)-u(x, t)) d y
$$

They show that these solutions (for a fixed initial condition) converge, when the kernel $J$ is appropriately rescaled, to the solution of the usual evolution problem for the $p$-Laplacian, $u_{t}=\Delta_{p} u$. In those references the case $p=1$ is also included. This particular case is more subtle than the case $p>1$, because of the appearance of $\frac{\nabla u}{|\nabla u|}$ as we pointed out before; note that there is also a similar difficulty even in (1.13) when $u(y)=u(x)$ for $x, y$ such that $x-y \in \operatorname{supp}(J)$. Furthermore, another difficulty when studying these nonlocal problems is to make sense to the boundary condition for general $h \in L^{1}(\partial \Omega)$, since it does not necessarily hold in the sense of traces. We will take advantage of the techniques developed in $[\mathbf{2}, \mathbf{4}]$ to obtain some of the results given here.

The paper is organized as follows: in the next section we prove the existence of variational solutions, hence the existence of weak solutions, of problem (1.5). In Section 3 we prove the characterization of both types of solutions given in Theorems 1.2 and 1.4; moreover we show that both concepts may not coincide. Finally, in Section 4 we prove that least gradient functions can be approximated by variational solutions of the nonlocal problems when the kernel $J$ is appropriately rescaled.

\section{The Dirichlet problem for the nonlocal 1-Laplacian}

The following Poincaré type inequality, established in [2], will be useful in the sequel.

Lemma 2.1. Given $q \geq 1, J: \mathbb{R}^{N} \rightarrow \mathbb{R}$ a nonnegative continuous radial function with compact support, $\Omega$ a bounded domain in $\mathbb{R}^{N}$, and $\psi \in$ $L^{q}\left(\Omega_{J} \backslash \bar{\Omega}\right)$, there exists $\lambda=\lambda(J, \Omega, q)>0$ such that

$\lambda \int_{\Omega}|u(x)|^{q} d x \leq \int_{\Omega} \int_{\Omega_{J}} J(x-y)\left|u_{\psi}(y)-u(x)\right|^{q} d y d x+\int_{\Omega_{J} \backslash \bar{\Omega}}|\psi(y)|^{q} d y$ for all $u \in L^{q}(\Omega)$.

Using ideas developed in $[\mathbf{2}, \mathbf{4}]$ we can prove the next two results. 
Theorem 2.2. Given $\psi \in L^{\infty}\left(\Omega_{J} \backslash \Omega\right)$ and $p>1$, there exists a variational solution to the homogeneous nonlocal p-Laplacian Dirichlet problem:

(2.1)

$$
\begin{cases}-\int_{\Omega_{J}} J(x-y)\left|\left(u_{p}\right)_{\psi}(y)-u_{p}(x)\right|^{p-2}\left(\left(u_{p}\right)_{\psi}(y)-u_{p}(x)\right) d y=0, & x \in \Omega, \\ u_{p}=\psi, & x \in \Omega_{J} \backslash \bar{\Omega} .\end{cases}
$$

Proof: Let us consider the functional

$$
\mathcal{F}_{p}(u):=\frac{1}{2 p} \int_{\Omega_{J}} \int_{\Omega_{J}} J(x-y)\left|u_{\psi}(y)-u_{\psi}(x)\right|^{p} d y d x, \quad u \in L^{p}(\Omega) .
$$

Set

$$
\theta:=\inf _{u \in L^{p}(\Omega)} \mathcal{F}_{p}(u)
$$

and let $\left\{u_{n}\right\}$ be a minimizing sequence. Then,

$$
\theta=\lim _{n \rightarrow \infty} \mathcal{F}_{p}\left(u_{n}\right) \quad \text { and } \quad K:=\sup _{n \in \mathbb{N}} \mathcal{F}_{p}\left(u_{n}\right)<+\infty .
$$

Poincaré type inequality (Lemma 2.1) yields

$$
\begin{aligned}
\lambda \int_{\Omega}\left|u_{n}(x)\right|^{p} d x \leq & \int_{\Omega} \int_{\Omega_{J}} J(x-y)\left|\left(u_{n}\right)_{\psi}(y)-u_{n}(x)\right|^{p} d y d x \\
& +\int_{\Omega_{J} \backslash \bar{\Omega}}|\psi(y)|^{p} d y \\
= & 2 p \mathcal{F}_{p}\left(u_{n}\right)+\int_{\Omega_{J} \backslash \bar{\Omega}}|\psi(y)|^{p} d y \leq 2 p K \\
& +\int_{\Omega_{J} \backslash \bar{\Omega}}|\psi(y)|^{p} d y .
\end{aligned}
$$

Therefore, we obtain that

$$
\int_{\Omega}\left|u_{n}(x)\right|^{p} d x \leq C, \quad \forall n \in \mathbb{N} .
$$

Hence, up to a subsequence, we have

$$
u_{n} \rightarrow u_{p} \quad \text { in } \quad L^{p}(\Omega) .
$$

Furthermore, using the weak lower semi-continuity of the functional $\mathcal{F}_{p}$, we get

$$
\mathcal{F}_{p}\left(u_{p}\right)=\inf _{u \in L^{p}(\Omega)} \mathcal{F}_{p}(u)
$$


Thus, given $\lambda>0$ and $w \in L^{p}(\Omega)$ (we extend it to $\Omega_{J} \backslash \Omega$ by zero), we have

$$
0 \leq \frac{\mathcal{F}_{p}\left(u_{p}+\lambda w\right)-\mathcal{F}_{p}\left(u_{p}\right)}{\lambda}
$$

or equivalently,

$$
\begin{array}{r}
0=\frac{1}{2} \int_{\Omega_{J}} \int_{\Omega_{J}} J(x-y)\left[\left|\left(u_{p}\right)_{\psi}(y)+\lambda w_{\psi}(y)-\left(\left(u_{p}\right)_{\psi}(x)+\lambda w_{\psi}(x)\right)\right|^{p}\right. \\
\left.-\left|\left(u_{p}\right)_{\psi}(y)-\left(u_{p}\right)_{\psi}(x)\right|^{p}\right] /(p \lambda) d y d x .
\end{array}
$$

Now, since $p>1$, we pass to the limit as $\lambda \downarrow 0$ to deduce

$$
\begin{array}{rl}
0 \leq \frac{1}{2} \int_{\Omega_{J}} \int_{\Omega_{J}} & J(x-y)\left|\left(u_{p}\right)_{\psi}(y)-\left(u_{p}\right)_{\psi}(x)\right|^{p-2} \\
& \times\left(\left(u_{p}\right)_{\psi}(y)-\left(u_{p}\right)_{\psi}(x)\right)\left((w)_{\psi}(y)-(w)_{\psi}(x)\right) d y d x .
\end{array}
$$

Taking $\lambda<0$ and proceeding as above we obtain the reverse inequality. Consequently, we conclude that

$$
\begin{aligned}
& 0=\frac{1}{2} \int_{\Omega_{J}} \int_{\Omega_{J}} J(x-y)\left|\left(u_{p}\right)_{\psi}(y)-\left(u_{p}\right)_{\psi}(x)\right|^{p-2} \\
& \times\left(\left(u_{p}\right)_{\psi}(y)-\left(u_{p}\right)_{\psi}(x)\right)\left((w)_{\psi}(y)-(w)_{\psi}(x)\right) d y d x \\
&=-\int_{\Omega_{J}} \int_{\Omega_{J}} J(x-y)\left|\left(u_{p}\right)_{\psi}(y)-\left(u_{p}\right)_{\psi}(x)\right|^{p-2} \\
& \times\left(\left(u_{p}\right)_{\psi}(y)-\left(u_{p}\right)_{\psi}(x)\right) d y(w)_{\psi}(x) d x .
\end{aligned}
$$

In particular, since $w=0$ in $\Omega_{J} \backslash \Omega$, it follows that

$0=-\int_{\Omega} \int_{\Omega_{J}} J(x-y)\left|\left(u_{p}\right)_{\psi}(y)-u_{p}(x)\right|^{p-2}\left(\left(u_{p}\right)_{\psi}(y)-u_{p}(x)\right) d y w(x) d x$,

which shows that $u_{p}$ is a solution of $(2.1)$.

Proposition 2.3. Let $u_{p}$ be the solution to (2.1) for $\psi \in L^{\infty}\left(\Omega_{J} \backslash \Omega\right)$. Then, $\left\|u_{p}\right\|_{\infty} \leq\|\psi\|_{\infty}$.

Proof: Set $M:=\|\psi\|_{\infty}$, multiply equation $(2.1)$ by $\left(u_{p}-M\right)^{+}$and integrate over $\Omega$ to obtain

$$
\begin{array}{rl}
0=-\int_{\Omega_{J}} \int_{\Omega_{J}} & J(x-y)\left|\left(u_{p}\right)_{\psi}(y)-\left(u_{p}\right)_{\psi}(x)\right|^{p-2} \\
& \times\left(\left(u_{p}\right)_{\psi}(y)-\left(u_{p}\right)_{\psi}(x)\right) d y\left(\left(u_{p}\right)_{\psi}-M\right)^{+}(x) d x
\end{array}
$$


or equivalently

$$
\begin{gathered}
0=\frac{1}{2} \int_{\Omega_{J}} \int_{\Omega_{J}} J(x-y)\left|\left(u_{p}\right)_{\psi}(y)-\left(u_{p}\right)_{\psi}(x)\right|^{p-2}\left(\left(u_{p}\right)_{\psi}(y)-\left(u_{p}\right)_{\psi}(x)\right) \\
\times\left(\left(\left(u_{p}\right)_{\psi}-M\right)^{+}(y)-\left(\left(u_{p}\right)_{\psi}-M\right)^{+}(x)\right) d y d x .
\end{gathered}
$$

In addition, since

$$
|r-s|^{p-2}(r-s)\left(r^{+}-s^{+}\right) \geq\left|r^{+}-s^{+}\right|^{p},
$$

it holds that

$$
\int_{\Omega_{J}} \int_{\Omega_{J}} J(x-y)\left|\left(\left(u_{p}\right)_{\psi}-M\right)^{+}(y)-\left(\left(u_{p}\right)_{\psi}-M\right)^{+}(x)\right|^{p} d y d x \leq 0 .
$$

Then, using again the Poincaré type inequality (Lemma 2.1) we get

$$
\int_{\Omega}\left|\left(u_{p}-M\right)^{+}(x)\right|^{p} d x=0
$$

This shows that $u_{p} \leq M$ a.e. in $\Omega$, for any $p>1$. Analogously, we can verify that $-M \leq u_{p}$ a.e. in $\Omega$. Thus $\left\|u_{p}\right\|_{\infty} \leq M$ for every $p>1$.

We are now ready to prove the existence of variational solutions to problem (1.5).

Theorem 2.4. Given $\psi \in L^{\infty}\left(\Omega_{J} \backslash \Omega\right)$ there exists a variational solution, hence a weak solution, to problem (1.5).

Proof: The previous result ensures that there exists a subsequence $p_{n} \rightarrow$ 1 , denoted by $p$, such that

$$
u_{p} \rightarrow u \quad \text { weakly in } \quad L^{1}(\Omega)
$$

and

$$
\begin{array}{r}
\left|\left(u_{p}\right)_{\psi}(y)-\left(u_{p}\right)_{\psi}(x)\right|^{p-2}\left(\left(u_{p}\right)_{\psi}(y)-\left(u_{p}\right)_{\psi}(x)\right) \rightarrow g(x, y) \\
\text { weakly in } L^{1}\left(\Omega_{J} \times \Omega_{J}\right) .
\end{array}
$$

The function $g$ is $L^{\infty}$-bounded by 1 , satisfies

$$
-\int_{\Omega_{J}} J(x-y) g(x, y) d y=0, \quad \text { a.e. } x \in \Omega,
$$

and, moreover, it is antisymmetric. In order to see that $J(x-y) g(x, y) \in J(x-y) \operatorname{sign}\left(u_{\psi}(y)-u_{\psi}(x)\right), \quad$ a.e. $(x, y) \in \Omega_{J} \times \Omega_{J}$, 
we need to prove that

$$
\begin{aligned}
-\int_{\Omega_{J}} \int_{\Omega_{J}} J & (x-y) g(x, y) d y u_{\psi}(x) d x \\
& =\frac{1}{2} \int_{\Omega_{J}} \int_{\Omega_{J}} J(x-y)\left|u_{\psi}(y)-u_{\psi}(x)\right| d y d x .
\end{aligned}
$$

In fact, it holds that

$$
\begin{gathered}
\frac{1}{2} \int_{\Omega_{J}} \int_{\Omega_{J}} J(x-y)\left|\left(u_{p}\right)_{\psi}(y)-\left(u_{p}\right)_{\psi}(x)\right|^{p} d y d x \\
=-\int_{\Omega_{J}} \int_{\Omega_{J}} J(x-y)\left|\left(u_{p}\right)_{\psi}(y)-\left(u_{p}\right)_{\psi}(x)\right|^{p-2} \\
\times\left(\left(u_{p}\right)_{\psi}(y)-\left(u_{p}\right)_{\psi}(x)\right) d y\left(u_{p}\right)_{\psi}(x) d x \\
=-\int_{\Omega_{J} \backslash \Omega} \int_{\Omega_{J}} J(x-y)\left|u_{p}(y)-u_{p}(x)\right|^{p-2} \\
\times\left(u_{p}(y)-u_{p}(x)\right) d y \psi(x) d x .
\end{gathered}
$$

Therefore,

$$
\begin{aligned}
\lim _{p} \frac{1}{2} \int_{\Omega_{J}} \int_{\Omega_{J}} J(x-y)\left|u_{p}(y)-u_{p}(x)\right|^{p} d y d x \\
=-\int_{\Omega_{J} \backslash \Omega} \int_{\Omega_{J}} J(x-y) g(x, y) d y \psi(x) d x \\
=-\int_{\Omega_{J}} \int_{\Omega_{J}} J(x-y) g(x, y) d y u_{\psi}(x) d x
\end{aligned}
$$

Now, by monotonicity (see for example [4, Lemma 6.29]), for all $\rho \in$ $L^{\infty}(\Omega)$,

$$
\begin{array}{rl}
-\int_{\Omega_{J}} \int_{\Omega_{J}} & J(x-y)\left|\rho_{\psi}(y)-\rho_{\psi}(x)\right|^{p-2} \\
& \times\left(\rho_{\psi}(y)-\rho_{\psi}(x)\right) d y\left(\left(u_{p}\right)_{\psi}(x)-\rho_{\psi}(x)\right) d x \\
\leq-\int_{\Omega_{J}} \int_{\Omega_{J}} J(x-y)\left|\left(u_{p}\right)_{\psi}(y)-\left(u_{p}\right)_{\psi}(x)\right|^{p-2} \\
\quad \times\left(\left(u_{p}\right)_{\psi}(y)-\left(u_{p}\right)_{\psi}(x)\right) d y\left(\left(u_{p}\right)_{\psi}(x)-\rho_{\psi}(x)\right) d x .
\end{array}
$$


Taking limits as $p \rightarrow 1$ and invoking (2.3) we get

$$
\begin{aligned}
& -\int_{\Omega_{J}} \int_{\Omega_{J}} J(x-y) \operatorname{sign}_{0}\left(\rho_{\psi}(y)-\rho_{\psi}(x)\right) d y\left(u_{\psi}(x)-\rho_{\psi}(x)\right) d x \\
\leq & -\int_{\Omega_{J}} \int_{\Omega_{J}} J(x-y) g(x, y) d y\left(u_{\psi}(x)-\rho_{\psi}(x)\right) d x,
\end{aligned}
$$

where

$$
\operatorname{sign}_{0}(z)= \begin{cases}1 & \text { if } z>0 \\ 0 & \text { if } z=0 \\ -1 & \text { if } z<0\end{cases}
$$

Taking $\rho=u \pm \lambda u, \lambda>0$, dividing by $\lambda$, and letting $\lambda \rightarrow 0$, we obtain (2.2), which finishes the proof.

\section{Characterization of the solutions of problem (1.5)}

Let us begin this section with the proof of Theorem 1.2, that characterizes weak solutions of (1.5). We will use the following notation: given $x \in \Omega$ we decompose $B_{1}(x)$ as

$$
\begin{aligned}
B_{1}(x) & =\left\{y \in B_{1}(x): u_{\psi}(y)>u(x)\right\} \cup\left\{y \in B_{1}(x): u_{\psi}(y)<u(x)\right\} \\
& \cup\left\{y \in B_{1}(x): u_{\psi}(y)=u(x)\right\} \\
& =: E_{+}^{x} \cup E_{-}^{x} \cup E_{0}^{x} .
\end{aligned}
$$

Hence

$$
1=\mu_{J}^{x}\left(E_{+}^{x}\right)+\mu_{J}^{x}\left(E_{-}^{x}\right)+\mu_{J}^{x}\left(E_{0}^{x}\right),
$$

and therefore, (3.1) and (3.2) below are equivalent:

$$
\begin{gathered}
-\mu_{J}^{x}\left(E_{0}^{x}\right) \leq \mu_{J}^{x}\left(E_{-}^{x}\right)-\mu_{J}^{x}\left(E_{+}^{x}\right) \leq \mu_{J}^{x}\left(E_{0}^{x}\right), \\
1 \leq 2\left(\mu_{J}^{x}\left(E_{+}^{x}\right)+\mu_{J}^{x}\left(E_{0}^{x}\right)\right) \text { and } 1 \leq 2\left(\mu_{J}^{x}\left(E_{-}^{x}\right)+\mu_{J}^{x}\left(E_{0}^{x}\right)\right) .
\end{gathered}
$$

That is,

$$
\begin{gathered}
(3.1) \equiv \mu_{J}^{x}\left(\left\{y \in B_{1}(x): u_{\psi}(y) \geq u(x)\right\}\right) \geq \frac{1}{2} \quad \text { and } \\
\mu_{J}^{x}\left(\left\{y \in B_{1}(x): u_{\psi}(y) \leq u(x)\right\}\right) \geq \frac{1}{2} .
\end{gathered}
$$

Proof of Theorem 1.2: Let $u$ be a weak solution to (1.5) with Dirichlet datum $\psi \in L^{1}\left(\Omega_{J} \backslash \bar{\Omega}\right)$, and take $g$ as in Definition 1.1. By (1.7) we have

$$
-\int_{B_{1}(x)} J(x-y) g(x, y) d y=0 .
$$


Thus,

$$
\begin{aligned}
0 & =\int_{E_{+}^{x}} J(x-y) g(x, y) d y+\int_{E_{-}^{x}} J(x-y) g(x, y) d y+\int_{E_{0}^{x}} J(x-y) g(x, y) d y \\
& =\mu_{J}^{x}\left(E_{+}^{x}\right)-\mu_{J}^{x}\left(E_{-}^{x}\right)+\int_{E_{0}^{x}} J(x-y) g(x, y) d y .
\end{aligned}
$$

Since $g \in[-1,1]$ in $E_{0}^{x}$, it holds that

$$
\mu_{J}^{x}\left(E_{-}^{x}\right)=\mu_{J}^{x}\left(E_{+}^{x}\right)+\int_{E_{0}^{x}} J(x-y) g(x, y) d y \leq \mu_{J}^{x}\left(E_{+}^{x}\right)+\mu_{J}^{x}\left(E_{0}^{x}\right)
$$

and

$$
\mu_{J}^{x}\left(E_{+}^{x}\right)=\mu_{J}^{x}\left(E_{-}^{x}\right)-\int_{E_{0}^{x}} J(x-y) g(x, y) d y \leq \mu_{J}^{x}\left(E_{-}^{x}\right)+\mu_{J}^{x}\left(E_{0}^{x}\right),
$$

that is

$$
-\mu_{J}^{x}\left(E_{0}^{x}\right) \leq \mu_{J}^{x}\left(E_{-}^{x}\right)-\mu_{J}^{x}\left(E_{+}^{x}\right) \leq \mu_{J}^{x}\left(E_{0}^{x}\right) .
$$

This proves, on account of (3.3), that $u$ satisfies the nonlocal median value property (1.8).

Let us show now that the converse is also true. Let $u$ be satisfying the nonlocal median value property (1.8), that is (on account of (3.3) again),

$$
-\mu_{J}^{x}\left(E_{0}^{x}\right) \leq \mu_{J}^{x}\left(E_{-}^{x}\right)-\mu_{J}^{x}\left(E_{+}^{x}\right) \leq \mu_{J}^{x}\left(E_{0}^{x}\right) .
$$

We have to find a function $g(x, y)$ verifying the conditions of Definition 1.1. For $x$ such that $\mu_{J}^{x}\left(E_{0}^{x}\right)=0$ let us define

$$
g(x, y):= \begin{cases}1 & \text { if } u_{\psi}(y)>u_{\psi}(x) \\ 0 & \text { if } u_{\psi}(y)=u_{\psi}(x) \\ -1 & \text { if } u_{\psi}(y)<u_{\psi}(x)\end{cases}
$$

and if $\mu_{J}^{x}\left(E_{0}^{x}\right)>0$,

$$
g(x, y):= \begin{cases}1 & \text { if } u_{\psi}(y)>u_{\psi}(x), \\ \frac{\mu_{J}^{x}\left(E_{-}^{x}\right)-\mu_{J}^{x}\left(E_{+}^{x}\right)}{\mu_{J}^{x}\left(E_{0}^{x}\right)} & \text { if } u_{\psi}(y)=u_{\psi}(x), \\ -1 & \text { if } u_{\psi}(y)<u_{\psi}(x) .\end{cases}
$$

This function $g$ belongs to $L^{\infty}$ and obviously $\|g\|_{\infty} \leq 1$. In addition, it verifies (1.6), that is,

$J(x-y) g(x, y) \in J(x-y) \operatorname{sign}\left(u_{\psi}(y)-u_{\psi}(x)\right), \quad$ a.e. $(x, y) \in \Omega_{J} \times \Omega_{J}$. 
Now, we have to check equation (1.7). In the case $\mu_{J}^{x}\left(E_{0}^{x}\right)=0$,

$$
\mu_{J}^{x}\left(E_{+}^{x}\right)=\mu_{J}^{x}\left(E_{-}^{x}\right)=\frac{1}{2},
$$

and we conclude that

$$
\begin{aligned}
\int_{B_{1}(x)} J(x-y) g(x, y) d y \\
=\int_{E_{+}^{x}} J(x-y) g(x, y) d y+\int_{E_{-}^{x}} J(x-y) g(x, y) d y \\
\quad+\int_{E_{0}^{x}} J(x-y) g(x, y) d y \\
=\int_{E_{+}^{x}} J(x-y) d y-\int_{E_{-}^{x}} J(x-y) d y=\mu_{J}^{x}\left(E_{+}^{x}\right)-\mu_{J}^{x}\left(E_{-}^{x}\right) \\
=\frac{1}{2}-\frac{1}{2}=0 .
\end{aligned}
$$

In the case $\mu_{J}^{x}\left(E_{0}^{x}\right)>0$,

$$
\begin{aligned}
& \int_{B_{1}(x)} J(x-y) g(x, y) d y \\
&=\int_{E_{+}^{x}} J(x-y) g(x, y) d y+\int_{E_{-}^{x}} J(x-y) g(x, y) d y \\
&+\int_{E_{0}^{x}} J(x-y) g(x, y) d y \\
&= \int_{E_{+}^{x}} J(x-y) d y-\int_{E_{-}^{x}} J(x-y) d y \\
&+\frac{\mu_{J}^{x}\left(E_{-}^{x}\right)-\mu_{J}^{x}\left(E_{+}^{x}\right)}{\mu_{J}^{x}\left(E_{0}^{x}\right)} \int_{E_{0}^{x}} J(x-y) d y \\
&= \mu_{J}^{x}\left(E_{+}^{x}\right)-\mu_{J}^{x}\left(E_{-}^{x}\right)+\left(\mu_{J}^{x}\left(E_{-}^{x}\right)-\mu_{J}^{x}\left(E_{+}^{x}\right)\right)=0 .
\end{aligned}
$$

This completes the proof.

Let us now characterize variational solutions as minimizers of $\mathcal{J}_{\psi}$.

Proof of Theorem 1.4: Let $u$ be a variational solution of problem (1.5). Then, there exists $g \in L^{\infty}\left(\Omega_{J} \times \Omega_{J}\right)$ with $\|g\|_{\infty} \leq 1$ verifying (1.10), (1.11), and (1.12). 
Given $w \in L^{1}(\Omega)$, multiplying (1.12) by $w(x)-u(x)$, integrating, and having in mind (1.11) and the antisymmetry of $g,(1.10)$, we get

$$
\begin{aligned}
0= & -\int_{\Omega_{J}} \int_{\Omega_{J}} J(x-y) g(x, y) d y\left(w_{\psi}(x)-u_{\psi}(x)\right) d x \\
= & \frac{1}{2} \int_{\Omega_{J}} \int_{\Omega_{J}} J(x-y) g(x, y)\left[\left(w_{\psi}(y)-w_{\psi}(x)\right)-\left(u_{\psi}(y)-u_{\psi}(x)\right)\right] d y d x \\
\leq & \frac{1}{2} \int_{\Omega_{J}} \int_{\Omega_{J}} J(x-y)\left|w_{\psi}(y)-w_{\psi}(x)\right| d y d x \\
& -\frac{1}{2} \int_{\Omega_{J}} \int_{\Omega_{J}} J(x-y)\left|u_{\psi}(y)-u_{\psi}(x)\right| d y d x \\
= & \mathcal{J}_{\psi}(w)-\mathcal{J}_{\psi}(u) .
\end{aligned}
$$

Therefore, $u$ is a minimizer of $\mathcal{J}_{\psi}$.

Assume now that $u$ minimizes the functional $\mathcal{J}_{\psi}$. Theorem 2.4 shows the existence of a variational solution $\bar{u}$ of (1.5). Namely, there exists $g: \Omega_{J} \times \Omega_{J} \rightarrow \mathbb{R}$ such that $g \in L^{\infty}\left(\Omega_{J} \times \Omega_{J}\right),\|g\|_{\infty} \leq 1, g(x, y)=$ $-g(y, x)$ for $(x, y)$ a.e. in $\Omega_{J} \times \Omega_{J}$,

(3.4) $J(x-y) g(x, y) \in J(x-y) \operatorname{sign}\left(\bar{u}_{\psi}(y)-\bar{u}_{\psi}(x)\right), \quad$ a.e. $(x, y) \in \Omega \times \Omega_{J}$,

and

$$
-\int_{\Omega_{J}} J(x-y) g(x, y) d y=0, \quad \text { a.e. } x \in \Omega .
$$

Since $u$ is a minimizer of $\mathcal{J}_{\psi}$,

$$
\mathcal{J}_{\psi}(\bar{u})-\mathcal{J}_{\psi}(u)=0
$$

On the other hand, arguing as in the other implication, we obtain that

$$
\begin{aligned}
0= & -\int_{\Omega_{J}} \int_{\Omega_{J}} J(x-y) g(x, y) d y\left(\bar{u}_{\psi}(x)-u_{\psi}(x)\right) d x \\
= & \frac{1}{2} \int_{\Omega_{J}} \int_{\Omega_{J}} J(x-y) g(x, y)\left[\left(\bar{u}_{\psi}(y)-\bar{u}_{\psi}(x)\right)-\left(u_{\psi}(y)-u_{\psi}(x)\right)\right] d y d x \\
= & \frac{1}{2} \int_{\Omega_{J}} \int_{\Omega_{J}} J(x-y)\left|\bar{u}_{\psi}(y)-\bar{u}_{\psi}(x)\right| d y d x \\
& -\frac{1}{2} \int_{\Omega_{J}} \int_{\Omega_{J}} J(x-y) g(x, y)\left(u_{\psi}(y)-u_{\psi}(x)\right) d y d x \\
= & \mathcal{J}_{\psi}(\bar{u})-\frac{1}{2} \int_{\Omega_{J}} \int_{\Omega_{J}} J(x-y) g(x, y)\left(u_{\psi}(y)-u_{\psi}(x)\right) d y d x .
\end{aligned}
$$


Therefore,

$$
\begin{aligned}
\frac{1}{2} \int_{\Omega_{J}} \int_{\Omega_{J}} J(x-y) g(x, y)\left(u_{\psi}(y)-u_{\psi}(x)\right) d y d x \\
\quad=\frac{1}{2} \int_{\Omega_{J}} \int_{\Omega_{J}} J(x-y)\left|u_{\psi}(y)-u_{\psi}(x)\right| d y d x
\end{aligned}
$$

Hence,

$J(x-y) g(x, y) \in J(x-y) \operatorname{sign}\left(u_{\psi}(y)-u_{\psi}(x)\right), \quad$ a.e. $(x, y) \in \Omega_{J} \times \Omega_{J}$, which jointly with (3.4) and (3.5) imply that $u$ is a variational solution to problem (1.5).

3.1. Examples. We conclude this section with some examples of weak solutions and variational solutions to the nonlocal 1-Laplacian, illustrating that both concepts may not coincide.

Example 3.1. Let us take $\Omega=(0,1)$, with Dirichlet datum an increasing function $\psi$, (for instance $\psi=0$ in $(-\infty, 0)$ and $\psi=1$ in $(1, \infty)$ ). Then, any increasing function between $\psi(0)$ and $\psi(1)$ is a variational solution to the nonlocal 1-Laplacian. Just observe that we can take $g$ as follows:

$$
g(x, y)= \begin{cases}1 & \text { if } y>x \\ 0 & \text { if } y=x \\ -1 & \text { if } y<x\end{cases}
$$

We note that an analogous argument shows that every nonincreasing function between its decreasing boundary data is a solution and also a solution to the local 1-Laplacian, $\left(\frac{u^{\prime}}{\left|u^{\prime}\right|}\right)^{\prime}=0$.

Example 3.2. There are weak solutions to the nonlocal 1-Laplacian that are not variational solutions. A simple example is the chessboard function $u: \Omega \subset \mathbb{R}^{2} \rightarrow \mathbb{R}$,

$$
u(x)=\left\{\begin{array}{ll}
a, & x \in Q_{2 i-1,2 j-1} \cap \Omega, \\
b, & x \in Q_{2 i, 2 j} \cap \Omega,
\end{array} \quad i, j \in \mathbb{N},\right.
$$

where each $Q_{i, j}$ is a square of size $\ell$ and we consider $a<b$ and $\ell \ll 1$. Indeed, let us fix some $\varepsilon \leq \ell$ and we just note that when $x$ is such that $u(x)=a$ we have

$$
\mu\left(\left\{y \in B_{\varepsilon}(x): u(y) \leq a\right\}\right)=\mu\left(\left\{y \in B_{\varepsilon}(x): u(y)=a\right\}\right) \geq \frac{1}{2} \mu\left(B_{\varepsilon}(x)\right),
$$

and

$$
\mu\left(\left\{y \in B_{\varepsilon}(x): u(y) \geq a\right\}\right)=\mu\left(B_{\varepsilon}(x)\right) \geq \frac{1}{2} \mu\left(B_{\varepsilon}(x)\right) .
$$


Moreover, when $x$ is such that $u(x)=b$ we have

$$
\mu\left(\left\{y \in B_{\varepsilon}(x): u(y) \leq b\right\}\right)=\mu\left(B_{\varepsilon}(x)\right) \geq \frac{1}{2} \mu\left(B_{\varepsilon}(x)\right),
$$

and

$$
\mu\left(\left\{y \in B_{\varepsilon}(x): u(y) \geq b\right\}\right)=\mu\left(\left\{y \in B_{\varepsilon}(x): u(y)=b\right\}\right) \geq \frac{1}{2} \mu\left(B_{\varepsilon}(x)\right) .
$$

On the other hand, we have that

$$
\mathcal{J}_{1}(u)>\mathcal{J}_{1}(1)
$$

In fact, $\mathcal{J}_{1}(u) \sim O(1)$ and $\mathcal{J}_{1}(1) \sim O(\ell)$ as $\ell \rightarrow 0$. Then, thanks to Theorem 1.4, $u$ is not a variational solution.

Remark 3.3. The above example shows that in general there is non uniqueness of weak solutions to the median value problem.

Other simple examples are the following:

Example 3.4. Let $\Omega=]-2,2[\times]-2,2[$, and choose $J$ supported in $B_{1}(0)$ and $\psi(x)=1$ if $\left.x \in\right]-2,2[\times(] 2,3[\cup]-3,-2[), \psi(x)=1$ if $x \in(] 2,3[\cup]-3,-2[) \times]-2,2[$ and $\psi(x)=0$ otherwise. In this case the constant function $u(x)=0$ in $\Omega$ is a weak solution to the nonlocal 1-Laplacian (any constant function between 0 and 1 is also a solution, though any constant function above 1 or below 0 is not). However, $u=0$ is not a variational solution by a similar argument to the above one. The function $u(x)=1$ is a variational solution.

In addition, if we rescale the support of $J$ to be the ball $B_{\varepsilon}(0)$, this function $u(x)=1$ is still a variational solution to the nonlocal 1-Laplacian for every $\varepsilon$ small enough. In the limit, $u=1$ is clearly the solution to the local 1-Laplacian with condition $u=1$ on the boundary of the square. See Section 4 for a general result of this nature.

Example 3.5. Consider the same domain as in the previous example, $\Omega=]-2,2[\times]-2,2\left[, J\right.$ supported in $B_{1}(0)$ and let now the boundary datum $\psi$ be given by: $\psi(x)=1$ if $x_{1}>0$ (here $x=\left(x_{1}, x_{2}\right)$ ), $\psi(x)=0$ if $x_{1}<0$. In this case the following function is a variational solution to the nonlocal 1-Laplacian: $u(x)=1$ if $x_{1}>0 ; u(x)=0$ if $x_{1}<0$. To see this fact, just take $g(x, y)=1$ if the vector $x y$ points upwards and -1 if it points downwards. 


\section{Local problems as limits of nonlocal ones: Convergence to functions of least gradient}

Let us start recalling that $u \in B V(\Omega)$ is called a function of least gradient if

$$
\int_{\Omega}|D u| \leq \int_{\Omega}|D(u+v)|
$$

for all $v \in B V(\Omega)$ such that $\operatorname{supp}(v) \subset \Omega$. It was proved in $[\mathbf{1 4}$, Theorem 2.2] that this definition is equivalent to require that

$$
\int_{\Omega}|D u| \leq \int_{\Omega}|D v|
$$

for all $v \in B V(\Omega)$ such that $\left.v\right|_{\partial \Omega}=\left.u\right|_{\partial \Omega}$.

In $[\mathbf{9}]$ it is studied the relation between functions of least gradient and 1-harmonic functions in the sense of (1.3). To this end, we need to introduce some preliminaries, see also $[\mathbf{3}, \mathbf{5}]$. If $w \in B V(\Omega)$ and $\zeta \in X_{N}(\Omega)$, given by

$$
X_{N}(\Omega)=\left\{\zeta \in L^{\infty}\left(\Omega, \mathbb{R}^{N}\right): \operatorname{div}(\zeta) \in L^{N}(\Omega)\right\},
$$

we can define the measure $(\zeta, D w): C_{0}^{\infty}(\Omega) \rightarrow \mathbb{R}$ by its action

$$
\langle(\zeta, D w), \varphi\rangle:=-\int_{\Omega} w \varphi \operatorname{div}(\zeta) d x-\int_{\Omega} w \zeta \cdot \nabla \varphi, \quad \forall \varphi \in C_{0}^{\infty}(\Omega) .
$$

Indeed $(\zeta, D w)$ is a Radon measure with finite total variation such that $(\zeta, D w)$ and $|(\zeta, D w)|$ are absolutely continuos with respect to the measure $|D w|$.

Moreover, in [5] a weak trace of the normal component of $\zeta \in X_{N}(\Omega)$ is defined on $\partial \Omega$. It is shown that there exists a linear operator $\gamma: X_{N}(\Omega) \rightarrow$ $L^{\infty}(\partial \Omega)$ such that for any $\zeta \in X_{N}(\Omega)$ it holds that $\|\gamma(\zeta)\|_{\infty} \leq\|\zeta\|_{\infty}$, and, if $\zeta \in C^{1}\left(\bar{\Omega}, \mathbb{R}^{N}\right)$,

$$
\gamma(\zeta)(x)=\zeta(x) \cdot \nu(x) \text { for all } x \in \partial \Omega,
$$

where $\nu(x)$ is the unit outward normal vector at $x \in \partial \Omega$. We will denote $\gamma(\zeta)(x)$ as $[\zeta, \nu](x)$.

In addition, it is proved the existence of a Green's formula, relating the function $[\zeta, \nu]$ and the measure $(\zeta, D w)$ as follows

$$
\int_{\Omega} w \operatorname{div}(\zeta) d x+\int_{\Omega}(\zeta, D w)=\int_{\partial \Omega}[\zeta, \nu] w \mathcal{H}^{N-1},
$$

for any $\zeta \in X_{N}(\Omega)$ and $w \in B V(\Omega)$. 
We are ready to define the following concept of solution of the Dirichlet problem

$$
\begin{cases}-\operatorname{div}\left(\frac{D u}{|D u|}\right)=0, & \text { in } \Omega, \\ u=\tilde{\psi}, & \text { on } \partial \Omega,\end{cases}
$$

with $\tilde{\psi} \in L^{1}(\partial \Omega)$. We say that $u \in B V(\Omega)$ is a solution of (4.1) if there exists $\zeta \in L^{\infty}\left(\Omega, \mathbb{R}^{N}\right)$, with $\|\zeta\|_{\infty} \leq 1$, satisfying

$$
\begin{aligned}
-\operatorname{div}(\zeta) & =0, \quad \text { in } \mathcal{D}^{\prime}(\Omega), \\
\int_{\Omega}(\zeta, D(u)) & =|D(u)|
\end{aligned}
$$

and

$$
[\zeta, \nu] \in \operatorname{sign}(\tilde{\psi}-u), \quad \mathcal{H}^{N-1} \text {-a.e. on } \partial \Omega .
$$

The following result was established in $[\mathbf{9}]$.

Theorem 4.1. Let $\tilde{\psi} \in L^{1}(\partial \Omega)$. Then, there exists a solution to (4.1). Moreover, for each $v \in B V(\Omega)$ satisfying $\left.v\right|_{\partial \Omega}=\tilde{\psi}$ the following conditions are equivalent:

(i) $v$ is a solution to (4.1).

(ii) $\Phi_{\tilde{\psi}}(v) \leq \Phi_{\tilde{\psi}}(u)$ for all $u \in B V(\Omega)$.

(iii) $v$ is a function of least gradient on $\Omega$ that equals $\tilde{\psi}$ on $\partial \Omega$.

Furthermore, it is shown that functions of least gradient may not be unique if the boundary datum $\tilde{\psi}$ is not continuous.

In the introduction we point out that (nonlocal) minimizers of $\mathcal{J}_{\psi}$ play the role of (local) minimizers of $\int_{\Omega}|D u|$. Now we prove Theorem 1.5 that jointly with Theorems 1.4 and 4.1 give the reason of such assertion.

Let us introduce the following notation: for a function $g$ defined in a set $D$, we define

$$
\bar{g}(x)= \begin{cases}g(x) & \text { if } x \in D, \\ 0 & \text { otherwise }\end{cases}
$$

Proof of Theorem 1.5: Given $\varepsilon>0$ small, we set $\Omega_{\varepsilon}:=\Omega_{J_{1, \varepsilon}}=\Omega+$ $\operatorname{supp}\left(J_{\varepsilon}\right)$. Then, there exists $g_{\varepsilon} \in L^{\infty}\left(\Omega_{\varepsilon} \times \Omega_{\varepsilon}\right), g_{\varepsilon}(x, y)=-g_{\varepsilon}(y, x)$ for almost all $(x, y) \in \Omega_{\varepsilon} \times \Omega_{\varepsilon},\left\|g_{\varepsilon}\right\|_{\infty} \leq 1$, such that

$$
\begin{aligned}
J\left(\frac{x-y}{\varepsilon}\right) g_{\varepsilon}(x, y) \in J\left(\frac{x-y}{\varepsilon}\right) \operatorname{sign}\left(\left(u_{\varepsilon}\right)_{\psi}(y)-\left(u_{\varepsilon}\right)_{\psi}(x)\right), & \text { a.e. }(x, y) \in \Omega_{J} \times \Omega_{J},
\end{aligned}
$$


and

$$
\int_{\Omega_{\varepsilon}} J\left(\frac{x-y}{\varepsilon}\right) g_{\varepsilon}(x, y) d y=0, \quad \text { a.e. } x \in \Omega \text {. }
$$

Set $M:=\|\psi\|_{L^{\infty}\left(\Omega_{J} \backslash \bar{\Omega}\right)}$. By $(4.2)$, we get

$$
\int_{\Omega_{\varepsilon}} \int_{\Omega_{\varepsilon}} J\left(\frac{x-y}{\varepsilon}\right) g_{\varepsilon}(x, y) d y\left(\left(u_{\varepsilon}\right)_{\psi}(x)-M\right)^{+} d x=0 .
$$

Then

$$
\begin{aligned}
0 & \leq \int_{\Omega_{\varepsilon}} \int_{\Omega_{\varepsilon}} J\left(\frac{x-y}{\varepsilon}\right)\left|\left(\left(u_{\varepsilon}\right)_{\psi}(y)-M\right)^{+}-\left(\left(u_{\varepsilon}\right)_{\psi}(x)-M\right)^{+}\right| d y d x \\
& =\int_{\Omega_{\varepsilon}} \int_{\Omega_{\varepsilon}} J\left(\frac{x-y}{\varepsilon}\right) g_{\varepsilon}(x, y)\left(\left(\left(u_{\varepsilon}\right)_{\psi}(y)-M\right)^{+}-\left(\left(u_{\varepsilon}\right)_{\psi}(x)-M\right)^{+}\right) d y d x \\
& =0 .
\end{aligned}
$$

Hence, from Poincaré type inequality, (Lemma 2.1), it follows that $u_{\varepsilon} \leq$ $M$ a.e., for all $\varepsilon>0$. Proceeding in a similar way we arrive to

$$
\left\|u_{\varepsilon}\right\|_{L^{\infty}(\Omega)} \leq M \text { for all } \varepsilon>0
$$

From here, we can assume that there exists a sequence $\varepsilon_{n} \rightarrow 0$ such that

$$
u_{\varepsilon_{n}} \rightarrow u \quad \text { weakly in } \quad L^{1}(\Omega)
$$

Using again (4.2), we obtain

$$
\int_{\Omega} \int_{\Omega_{\varepsilon}} J\left(\frac{x-y}{\varepsilon}\right) g_{\varepsilon}(x, y) d y u_{\varepsilon}(x) d x=0 .
$$

Furthermore,

$$
\begin{aligned}
\int_{\Omega_{\varepsilon}} \int_{\Omega_{\varepsilon}} J & \left(\frac{x-y}{\varepsilon}\right) g_{\varepsilon}(x, y) d y\left(u_{\varepsilon}\right)_{\psi}(x) d x \\
= & \int_{\Omega_{\varepsilon} \backslash \bar{\Omega}} \int_{\Omega_{\varepsilon}} J\left(\frac{x-y}{\varepsilon}\right) g_{\varepsilon}(x, y) d y \psi(x) d x .
\end{aligned}
$$


Now,

$$
\begin{aligned}
\left|\frac{1}{\varepsilon^{1+N}} \int_{\Omega_{\varepsilon} \backslash \bar{\Omega}} \int_{\Omega_{\varepsilon}} J\left(\frac{x-y}{\varepsilon}\right) g_{\varepsilon}(x, y) d y \psi(x) d x\right| \\
\leq \frac{1}{\varepsilon^{1+N}} \int_{\Omega_{\varepsilon} \backslash \bar{\Omega}} \int_{\Omega_{\varepsilon}} J\left(\frac{x-y}{\varepsilon}\right) d y|\psi(x)| d x \\
\leq \frac{1}{\varepsilon} M \int_{\Omega_{\varepsilon} \backslash \bar{\Omega}}\left(\frac{1}{\varepsilon^{N}} \int_{\Omega_{\varepsilon}} J\left(\frac{x-y}{\varepsilon}\right) d y\right) d x \\
\leq \frac{1}{\varepsilon} M\left|\Omega_{\varepsilon} \backslash \Omega\right| \leq M_{1} .
\end{aligned}
$$

Consequently, integrating by parts in (4.3),

$$
\frac{1}{\varepsilon^{N+1}} \int_{\Omega_{\varepsilon}} \int_{\Omega_{\varepsilon}} J\left(\frac{x-y}{\varepsilon}\right)\left|\left(u_{\varepsilon}\right)_{\psi}(y)-\left(u_{\varepsilon}\right)_{\psi}(x)\right| d y d x \leq 2 M_{1} .
$$

Let us compute,

$$
\begin{aligned}
\int_{\Omega_{J}} \int_{\Omega_{J}} J & \left(\frac{x-y}{\varepsilon}\right)\left|\left(u_{\varepsilon}\right)_{\psi}(y)-\left(u_{\varepsilon}\right)_{\psi}(x)\right| d y d x \\
= & \int_{\Omega_{\varepsilon}} \int_{\Omega_{\varepsilon}} J\left(\frac{x-y}{\varepsilon}\right)\left|\left(u_{\varepsilon}\right)_{\psi}(y)-\left(u_{\varepsilon}\right)_{\psi}(x)\right| d y d x \\
& +2 \int_{\Omega_{\varepsilon}} \int_{\Omega_{J} \backslash \bar{\Omega}_{\varepsilon}} J\left(\frac{x-y}{\varepsilon}\right)\left|\left(u_{\varepsilon}\right)_{\psi}(y)-\left(u_{\varepsilon}\right)_{\psi}(x)\right| d y d x \\
& +\int_{\Omega_{J} \backslash \bar{\Omega}_{\varepsilon}} \int_{\Omega_{J} \backslash \bar{\Omega}_{\varepsilon}} J\left(\frac{x-y}{\varepsilon}\right)\left|\left(u_{\varepsilon}\right)_{\psi}(y)-\left(u_{\varepsilon}\right)_{\psi}(x)\right| d y d x
\end{aligned}
$$

Now, since $\psi \in W^{1,1}\left(\Omega_{J} \backslash \bar{\Omega}\right)$, we get

$$
\begin{aligned}
& \frac{1}{\varepsilon^{N+1}} \int_{\Omega_{J} \backslash \bar{\Omega}_{\varepsilon}} \int_{\Omega_{J} \backslash \bar{\Omega}_{\varepsilon}} J\left(\frac{x-y}{\varepsilon}\right)\left|\left(u_{\varepsilon}\right)_{\psi}(y)-\left(u_{\varepsilon}\right)_{\psi}(x)\right| d y d x \\
& \quad=\int_{\Omega_{J} \backslash \bar{\Omega}_{\varepsilon}} \int_{\Omega_{J} \backslash \bar{\Omega}_{\varepsilon}} \frac{1}{\varepsilon^{N}} J\left(\frac{x-y}{\varepsilon}\right) \frac{|\psi(y)-\psi(x)|}{\varepsilon} d y d x \leq M_{2}
\end{aligned}
$$


On the other hand, we have

$$
\begin{aligned}
& \frac{1}{\varepsilon^{N+1}} \int_{\Omega_{J} \backslash \bar{\Omega}_{\varepsilon}} \int_{\Omega_{\varepsilon}} J\left(\frac{x-y}{\varepsilon}\right)\left|\left(u_{\varepsilon}\right)_{\psi}(y)-\left(u_{\varepsilon}\right)_{\psi}(x)\right| d y d x \\
& \quad=\int_{\Omega_{J} \backslash \bar{\Omega}_{\varepsilon}} \int_{\Omega_{\varepsilon} \backslash \bar{\Omega}} \frac{1}{\varepsilon^{N}} J\left(\frac{x-y}{\varepsilon}\right) \frac{|\psi(y)-\psi(x)|}{\varepsilon} d y d x \leq M_{3} .
\end{aligned}
$$

Furthermore, from (4.5), (4.4), (4.6), and (4.7), it follows that

$$
\frac{1}{\varepsilon^{N+1}} \int_{\Omega_{J}} \int_{\Omega_{J}} J\left(\frac{x-y}{\varepsilon}\right)\left|\left(u_{\varepsilon}\right)_{\psi}(y)-\left(u_{\varepsilon}\right)_{\psi}(x)\right| d y d x \leq M_{4},
$$

or equivalently,

$$
\int_{\Omega_{J}} \int_{\Omega_{J}} J\left(\frac{x-y}{\varepsilon}\right)\left|\frac{\left(u_{\varepsilon}\right)_{\psi}(y)-\left(u_{\varepsilon}\right)_{\psi}(x)}{\varepsilon}\right| d x d y \leq M_{4} \varepsilon^{N}, \quad \forall n \in \mathbb{N} .
$$

Invoking [4, Theorem 6.11], there exists a subsequence, still denoted as $u_{\varepsilon_{n}}$, and a function $w \in B V\left(\Omega_{J}\right)$ such that

$$
\left(u_{\varepsilon_{n}}\right)_{\psi} \rightarrow w \quad \text { strongly in } \quad L^{1}\left(\Omega_{J}\right)
$$

and

$$
J(z) \chi_{\Omega}\left(\cdot+\varepsilon_{n} z\right) \frac{\left(u_{\varepsilon_{n}}\right)_{\psi}\left(\cdot+\varepsilon_{n} z\right)-\left(u_{\varepsilon_{n}}\right)_{\psi}(\cdot)}{\varepsilon_{n}} \rightarrow J(z) z \cdot D w
$$

weakly as measures. Hence, it is easy to obtain that

$$
w(x)=u_{\psi}(x)= \begin{cases}u(x), & \text { in } x \in \Omega, \\ \psi(x), & \text { in } x \in \Omega_{J} \backslash \bar{\Omega}\end{cases}
$$

and that $u \in B V(\Omega)$.

Moreover, we can also assume that

$$
J(z) \chi_{\Omega_{J}}\left(x+\varepsilon_{n} z\right) \bar{g}_{\varepsilon_{n}}\left(x, x+\varepsilon_{n} z\right) \rightarrow \Lambda(x, z)
$$

weakly* in $L^{\infty}\left(\Omega_{J}\right) \times L^{\infty}\left(\mathbb{R}^{N}\right)$ for some function $\Lambda \in L^{\infty}\left(\Omega_{J}\right) \times L^{\infty}\left(\mathbb{R}^{N}\right)$, $\Lambda(x, z) \leq J(z)$ almost every where in $\Omega_{J} \times \mathbb{R}^{N}$. Take $v \in \mathcal{D}(\Omega)$, and $\varepsilon=\varepsilon_{n}$ small enough, by $(4.2)$,

$$
\int_{\Omega} \int_{\Omega} J\left(\frac{x-y}{\varepsilon_{n}}\right) g_{\varepsilon_{n}}(x, y) v(x) d y d x=0 .
$$


Then, integrating by parts,

$$
\begin{aligned}
0 & =\frac{1}{2} \int_{\Omega} \int_{\Omega} J\left(\frac{x-y}{\varepsilon_{n}}\right) g_{\varepsilon_{n}}(x, y)(v(y)-v(x)) d y d x \\
& =\frac{1}{2} \int_{\Omega} \int_{\mathbb{R}^{N}} J\left(\frac{x-y}{\varepsilon_{n}}\right) \chi_{\Omega}(y) \bar{g}_{\varepsilon_{n}}(x, y)(v(y)-v(x)) d y d x .
\end{aligned}
$$

Changing now variables, and applying Fubini's Theorem,

$$
\int_{\mathbb{R}^{N}} \int_{\Omega} J(z) \chi_{\Omega}\left(x+\varepsilon_{n} z\right) \bar{g}_{\varepsilon_{n}}\left(x, x+\varepsilon_{n} z\right) \frac{\bar{v}\left(x+\varepsilon_{n} z\right)-v(x)}{\varepsilon_{n}} d x d z=0 .
$$

By (4.9), passing to the limit in (4.10), we get

$$
\int_{\mathbb{R}^{N}} \int_{\Omega} \Lambda(x, z) z \cdot \nabla v(x) d x d z=0
$$

for all $v \in \mathcal{D}(\Omega)$. We set $\zeta=\left(\zeta_{1}, \ldots, \zeta_{N}\right)$, the vector field defined by

$$
\zeta_{i}(x):=\frac{1}{C_{J}} \int_{\mathbb{R}^{N}} \Lambda(x, z) z_{i} d z, \quad i=1, \ldots, N,
$$

where $C_{J}:=\int_{\mathbb{R}^{N}} J(z)\left|z_{N}\right| d z$. Then, $\zeta \in L^{\infty}\left(\Omega_{J}, \mathbb{R}^{N}\right)$, and from (4.11),

$$
-\operatorname{div}(\zeta)=0 \quad \text { in } \quad \mathcal{D}^{\prime}(\Omega)
$$

Let us see that

$$
\|\zeta\|_{L^{\infty}\left(\Omega_{J}\right)} \leq 1
$$

Given $\xi \in \mathbb{R}^{N} \backslash\{0\}$, let $R_{\xi}$ be the rotation such that, for its transpose, $R_{\xi}^{\text {trans }}(\xi)=\mathbf{e}_{1}|\xi|$. If we make the change of variables $z=R_{\xi}(y)$, we obtain

$$
\begin{aligned}
\zeta(x) \cdot \xi & =\frac{1}{C_{J}} \int_{\mathbb{R}^{N}} \Lambda(x, z) z \cdot \xi d z=\frac{1}{C_{J}} \int_{\mathbb{R}^{N}} \Lambda\left(x, R_{\xi}(y)\right) R_{\xi}(y) \cdot \xi d y \\
& =\frac{1}{C_{J}} \int_{\mathbb{R}^{N}} \Lambda\left(x, R_{\xi}(y)\right) y_{1}|\xi| d y .
\end{aligned}
$$

On the other hand, since $J$ is a radial function and $\Lambda(x, z) \leq J(z)$ almost everywhere, we have

$$
C_{J}=\int_{\mathbb{R}^{N}} J(z)\left|z_{1}\right| d z
$$


and

$$
|\zeta(x) \cdot \xi| \leq \frac{1}{C_{J}} \int_{\mathbb{R}^{N}} J(y)\left|y_{1}\right| d y|\xi|=|\xi|, \quad \text { a.e. } x \in \Omega_{J} .
$$

Therefore, $\|\zeta\|_{L^{\infty}\left(\Omega_{J}\right)} \leq 1$.

To finish the proof, we only need to prove that

$$
(\zeta, D u)=|D u| \quad \text { as measures in } \Omega
$$

and

$$
[\zeta, \nu] \in \operatorname{sign}(\tilde{\psi}-u), \quad \mathcal{H}^{N-1} \text {-a.e. on } \partial \Omega \text {. }
$$

Let us take $w_{m} \in W^{1,1}(\Omega) \cap C(\Omega)$ such that $w_{m}=\tilde{\psi} \mathcal{H}^{N-1}$-a.e. on $\partial \Omega$, and $w_{m} \rightarrow u$ in $L^{1}(\Omega)$. Set $v_{m, n}:=\left(u_{\varepsilon_{n}}\right)_{\psi}-\left(w_{m}\right)_{\psi}$. By $(4.2)$,

$$
\begin{aligned}
0 & =-\frac{1}{\varepsilon_{n}{ }^{N+1}} \int_{\Omega_{J}} \int_{\Omega_{J}} J\left(\frac{x-y}{\varepsilon_{n}}\right) g_{\varepsilon_{n}}(x, y) v_{m, n}(x) d y d x \\
& =\frac{1}{2 \varepsilon_{n}^{N+1}} \int_{\Omega_{J}} \int_{\Omega_{J}} J\left(\frac{x-y}{\varepsilon_{n}}\right) g_{\varepsilon_{n}}(x, y)\left(v_{m, n}(y)-v_{m, n}(x)\right) d y d x \\
& =H_{n}^{1}+H_{m, n}^{1}
\end{aligned}
$$

where

$$
H_{n}^{1}=\frac{1}{2} \int_{\Omega_{J}} \int_{\mathbb{R}^{N}} J(z) \chi_{\Omega_{J}}\left(x+\varepsilon_{n} z\right)\left|\frac{\left(u_{\varepsilon_{n}}\right)_{\psi}\left(x+\varepsilon_{n} z\right)-\left(u_{\varepsilon_{n}}\right)_{\psi}(x)}{\varepsilon_{n}}\right| d z d x
$$

and

$$
\begin{aligned}
H_{m, n}^{2}=-\frac{1}{2} \int_{\Omega_{J}} \int_{\mathbb{R}^{N}} J(z) \chi_{\Omega_{J}}\left(x+\varepsilon_{n} z\right) \bar{g}_{\varepsilon_{n}}\left(x, x+\varepsilon_{n} z\right) \\
\times \frac{\left(w_{m}\right)_{\psi}\left(x+\varepsilon_{n} z\right)-\left(w_{m}\right)_{\psi}(x)}{\varepsilon_{n}} d z d x .
\end{aligned}
$$

Taking into account (4.8), we get

$$
\begin{aligned}
\liminf _{n \rightarrow \infty} H_{n}^{1} \geq \frac{C_{J}}{2} \int_{\Omega_{J}}\left|D u_{\psi}\right|= & \frac{C_{J}}{2} \int_{\Omega}|D u| \\
& +\frac{C_{J}}{2} \int_{\partial \Omega}|u-\tilde{\psi}| d \mathcal{H}^{N-1}+\frac{C_{J}}{2} \int_{\Omega_{J} \backslash \bar{\Omega}}|\nabla \psi| .
\end{aligned}
$$


On the other hand, since $\left(w_{m}\right)_{\psi} \in W^{1,1}\left(\Omega_{J}\right)$, by (4.9),

$$
\begin{aligned}
\lim _{n \rightarrow \infty} H_{m, n}^{2} & =-\frac{1}{2} \int_{\Omega_{J}} \int_{\mathbb{R}^{N}} \Lambda(x, z) z \cdot \nabla\left(w_{m}\right)_{\psi}(x) d z d x \\
& =-\frac{C_{J}}{2} \int_{\Omega_{J}} \zeta(x) \cdot \nabla\left(w_{m}\right)_{\psi}(x) d x
\end{aligned}
$$

Consequently, taking $n \rightarrow \infty$ in (4.14), we obtain

$$
\begin{aligned}
0 \geq \int_{\Omega}|D u|+\int_{\partial \Omega}|u-\tilde{\psi}| d \mathcal{H}^{N-1} & +\int_{\Omega_{J} \backslash \bar{\Omega}}|\nabla \psi| \\
& -\int_{\Omega_{J}} \zeta(x) \cdot \nabla\left(w_{m}\right)_{\psi}(x) d x
\end{aligned}
$$

Now,

$$
\begin{aligned}
-\int_{\Omega_{J}} \zeta(x) \cdot \nabla\left(w_{m}\right)_{\psi}(x) d x= & -\int_{\Omega} \zeta(x) \cdot \nabla w_{m}(x) d x \\
& -\int_{\Omega_{J} \backslash \bar{\Omega}} \zeta(x) \cdot \nabla \psi(x) d x \\
= & \int_{\Omega} \operatorname{div} \zeta(x) w_{m}(x) d x-\int_{\partial \Omega}[\zeta, \nu] \tilde{\psi} d \mathcal{H}^{N-1} \\
& -\int_{\Omega_{J} \backslash \bar{\Omega}} \zeta(x) \cdot \nabla \psi(x) d x .
\end{aligned}
$$

Since

$$
\int_{\Omega_{J} \backslash \bar{\Omega}}|\nabla \psi|-\int_{\Omega_{J} \backslash \bar{\Omega}} \zeta(x) \cdot \nabla \psi(x) d x \geq 0
$$

from (4.15), we have

$$
0 \geq \int_{\Omega}|D u|+\int_{\partial \Omega}|u-\tilde{\psi}| d \mathcal{H}^{N-1}+\int_{\Omega} \operatorname{div} \zeta(x) w_{m}(x) d x-\int_{\partial \Omega}[\zeta, \nu] \tilde{\psi} d \mathcal{H}^{N-1}
$$


Letting $m \rightarrow \infty$ and using Green's formula, we deduce

$$
\begin{aligned}
0 \geq & \int_{\Omega}|D u|+\int_{\partial \Omega}|u-\tilde{\psi}| d \mathcal{H}^{N-1}+\int_{\Omega} \operatorname{div} \zeta(x) u(x) d x \\
& -\int_{\partial \Omega}[\zeta, \nu] \tilde{\psi} d \mathcal{H}^{N-1} \\
= & \int_{\Omega}|D u|+\int_{\partial \Omega}|u-\tilde{\psi}| d \mathcal{H}^{N-1}-\int_{\Omega}(\zeta, D u)+\int_{\partial \Omega}[\zeta, \nu] u d \mathcal{H}^{N-1} \\
& -\int_{\partial \Omega}[\zeta, \nu] \tilde{\psi} d \mathcal{H}^{N-1} .
\end{aligned}
$$

Furthermore, since $|(\zeta, D u)| \leq|D u|$ and $|[\zeta, \nu]| \leq 1$,

$$
\begin{aligned}
\int_{\partial \Omega}|u-\tilde{\psi}| d \mathcal{H}^{N-1} & \leq \int_{\Omega}(\zeta, D u)-\int_{\Omega}|D u|+\int_{\partial \Omega}[\zeta, \nu](\tilde{\psi}-u) d \mathcal{H}^{N-1} \\
& \leq \int_{\partial \Omega}|u-\tilde{\psi}| d \mathcal{H}^{N-1} .
\end{aligned}
$$

Therefore (4.12) and (4.13) are satisfied and the proof is finished.

Remark 4.2. Sternberg, Williams, and Ziemer proved in [13] that, for a bounded Lipschitz domain $\Omega \subset \mathbb{R}^{N}$ such that $\partial \Omega$ has non-negative mean curvature (in a weak sense) and is not locally area-minimizing, and for $h \in C(\partial \Omega)$, there exists a unique function of least gradient $u \in B V(\Omega) \cap$ $C(\bar{\Omega})$ such that $u=h$ on $\partial \Omega$. Therefore, as consequence of Theorems 4.1 and 1.5, we have that, assuming the continuity of the boundary data and the above conditions on the domain, we can approximate the function of least gradient by a sequence of variational solutions of the nonlocal problem (1.5).

Acknowledgements. J. M. Mazón and J. Toledo are supported by the Spanish project MTM2012-31103, and M. Pérez-Llanos and J. D. Rossi are partially supported by the Spanish project MTM2013-40846-P.

\section{References}

[1] F. Andreu, C. Ballester, V. Caselles, and J. M. Mazón, The Dirichlet problem for the total variation flow, J. Funct. Anal. 180(2) (2001), 347-403. DOI: 10.1006/jfan.2000.3698. 
[2] F. Andreu, J. M. Mazón, J. D. Rossi, And J. Toledo, A nonlocal $p$-Laplacian evolution equation with nonhomogeneous Dirichlet boundary conditions, SIAM J. Math. Anal. 40(5) (2008/09), 1815-1851. DOI : 10.1137/080720991.

[3] F. Andreu-Vaillo, V. Caselles, and J. M. Mazón, "Parabolic Quasilinear Equations Minimizing Linear Growth Functionals", Progress in Mathematics 223, Birkhäuser Verlag, Basel, 2004. DOI: $10.1007 / 978-3-0348-7928-6$.

[4] F. Andreu-Vaillo, J. M. Mazón, J. D. Rossi, and J. ToledoMelero, "Nonlocal Diffusion Problems", Mathematical Surveys and Monographs 165, American Mathematical Society, Providence, RI; Real Sociedad Matemática Española, Madrid, 2010. DOI: $10.1090 /$ surv/165.

[5] G. Anzellotti, Pairings between measures and bounded functions and compensated compactness, Ann. Mat. Pura Appl. (4) 135(1) (1983), 293-318. DOI: 10.1007/BF01781073.

[6] E. Bombieri, E. De Giorgi, And E. Giusti, Minimal cones and the Bernstein problem, Invent. Math. 7(3) (1969), 243-268. DOI: 10.1007/BF01404309.

[7] D. Hartenstine And M. Rudd, Asymptotic statistical characterizations of $p$-harmonic functions of two variables, Rocky Mountain J. Math. 41(2) (2011), 493-504. DOI: 10.1216/RMJ-2011-41-2-493.

[8] J. J. Manfredi, M. Parviainen, and J. D. Rossi, An asymptotic mean value characterization for $p$-harmonic functions, Proc. Amer. Math. Soc. 138(3) (2010), 881-889. DOI: 10.1090/S00029939-09-10183-1.

[9] J. M. Mazón, J. D. Rossi, And S. Segura de León, Functions of least gradient and 1-harmonic functions, Indiana Univ. Math. J. 63(4) (2014), 1067-1084. DOI: 10.1512/iumj.2014.63.5327.

[10] S. G. NoAh, The median of a continuous function, Real Anal. Exchange 33(1) (2007/2008), 269-274.

[11] M. B. RuDD, Statistical exponential formulas for homogeneous diffusion, Commun. Pure Appl. Anal. 14(1) (2015), 269-284. DOI: 10.3934/cpaa.2015.14.269.

[12] M. B. Rudd And H. A. VAn Dyke, Median values, 1-harmonic functions, and functions of least gradient, Commun. Pure Appl. Anal. 12(2) (2013), 711-719. DOI: 10.3934/cpaa.2013.12.711.

[13] P. Sternberg, G. Williams, and W. P. Ziemer, Existence, uniqueness, and regularity for functions of least gradient, J. Reine Angew. Math. 430 (1992), 35-60. DOI: 10.1515/crll.1992.430.35. 
[14] P. Sternberg and W. P. Ziemer, The Dirichlet problem for functions of least gradient, in: "Degenerate Diffusions" (Minneapolis, MN, 1991), IMA Vol. Math. Appl. 47, Springer, New York, 1993, pp. 197-214. DOI: 10.1007/978-1-4612-0885-3_14.

J. M. Mazón and J. Toledo:

Departament d'Anàlisi Matemàtica

Universitat de València

Dr. Moliner 50

46100 Burjassot

Spain

E-mail address: mazon@uv.es

E-mail address: toledojj@uv.es

Mayte Pérez-Llanos:

Departamento de Matemáticas

Universidad Autónoma de Madrid

Facultad de Ciencias, mod 17

Campus de Cantoblanco

28049 Madrid

Spain

E-mail address: mayte.perez@uam.es

J. D. Rossi:

Departamento de Matemática

FCEyN UBA

Ciudad Universitaria, Pab 1 (1428)

Buenos Aires

Argentina

E-mail address: jrossi@dm.uba.ar

Primera versió rebuda el 5 de març de 2014,

darrera versió rebuda el 29 d'agost de 2014. 